\title{
Evaluation of Pharmacist Involvement in Outpatient Transitions of Care
}

\author{
Adam S. Whalley, PharmD', Marissa C. Salvo, PharmD, BCACP², Susan F. Levine, MD, MPH', \\ Kevin W. Chamberlin, PharmD, FASCP 2,4 , and Danielle Beaudoin, CMA, AAMA ${ }^{3}$ \\ 1, Portland, USA; ${ }^{2}$ School of Pharmacy, UConn, Storrs, CT, USA; ${ }^{3}$ General Medicine Associates, UConn Health, Farmington, CT, USA; ${ }^{4}$ UConn John \\ Dempsey Hospital, UConn Health, Farmington, CT, USA.
}

KEY WORDS: transitions of care; pharmacy; medication reconciliation; outpatient; general medicine.

$\mathrm{J}$ Gen Intern Med 33(8):1227-8

DOI: $10.1007 / \mathrm{s} 11606-018-4459-\mathrm{x}$

(c) Society of General Internal Medicine 2018

\section{INTRODUCTION}

Approximately $20 \%$ of patients will experience an adverse event during the transition from hospital to home, with two thirds of the events due to medications. ${ }^{1}$ Medication reconciliation at transitions can minimize and/or eliminate the risk of medication errors by ensuring each patient's regimen is accurate and updated. ${ }^{2}$ Pharmacists are uniquely qualified to complete this task due to their expertise in assessing patients' medication profiles, identifying medication errors, and providing recommendations for resolution. Studies have consistently found that pharmacist involvement in medication reconciliation in the inpatient setting reduces medication errors. ${ }^{3,4}$ However, few have examined the integration of a pharmacist in the outpatient setting, following a patient's discharge to home.

UConn Health's academic hospital (JDH) utilizes trained pharmacy technicians to conduct comprehensive medication history taking. At discharge, medication reconciliation is performed by a physician, with any changes noted in the discharge summary. General Medicine Associates (GMA) is a primary care clinic within the UConn Health system that includes 49 general medicine providers, 34 of whom are medical residents, and a pharmacy team (faculty, residents, and students). While the facilities utilize separate medical records, providers have access to both.

Our objective was to assess the impact of pharmacist involvement on the identification and resolution of medication discrepancies during post-discharge follow-up appointments

The retrospective results and study's methods were presented at the American Society of Health Systems Pharmacists' 2016 Midyear Clinical Meeting.

Published online April 30, 2018 at GMA. The institution's investigational review board (IRB) granted exemption to this project, as it was determined to be a quality improvement project.

\section{METHODS}

A retrospective and prospective analysis was completed to determine the impact of pharmacist integration into an outpatient transition of care process. The retrospective review included 31 patients discharged from JDH who had a Medicare transition of care management (TCM) visit between February 2016 and September 2016. The prospective review included 14 patients discharged from hospital to home with a scheduled follow-up/TCM appointment between October 2016 and March 2017.

In both cohorts, a medical assistant (MA) called the patient prior to the scheduled appointment to review the discharge summary and conduct medication reconciliation. Following, a pharmacist reviewed the discharge instructions, medication list, and the MA's medication reconciliation and compared this with the outpatient medication profile to identify medication discrepancies and provide recommendations to the primary care provider (PCP).

Discrepancies were identified as one of the five categories listed in Table 1 and severity was established based on the type and medication involved in the discrepancy. A two-pharmacist consensus on severity was established utilizing the Institute of Safe Medication Practices high-alert medications, previously published literature on medication discrepancies, and clinical judgment. ${ }^{5}$ Following the patient's discharge appointment, the physician's note was reviewed to determine the number and percentage of medication discrepancies addressed.

\section{RESULTS}

The patient population was similar between the two cohorts with respect to age $(p=0.74)$, gender $(p=0.45)$, and number of medications at discharge $(12.8 \pm 5.4$ vs. $13.1 \pm 7.1 ; p=0.9)$. 
Table 1 Types of medication discrepancies identified

\begin{tabular}{ll}
\hline \hline Discrepancy & Definition \\
\hline Omission & $\begin{array}{l}\text { Medications active in outpatient profile not included in } \\
\text { discharge instructions }\end{array}$ \\
Addition & $\begin{array}{l}\text { Medications included in discharge instructions not } \\
\text { currently active in outpatient profile }\end{array}$ \\
Dose change & $\begin{array}{l}\text { Medications active in outpatient profile and included in } \\
\text { discharge instruction, but with a change in dose, } \\
\text { frequency, or directions }\end{array}$ \\
Interaction & $\begin{array}{l}\text { Drug interaction deemed potentially significant } \\
\text { Optimization } \\
\text { zation, and all other discrepancies not included in above } \\
\text { categories }\end{array}$ \\
\hline
\end{tabular}

The number of medication discrepancies identified was not significantly different $(p=0.09)$ between the retrospective (11.3, SD 5.8) and prospective (12, SD 7.8) cohorts. Of the identified medication discrepancies, $21 \%$ involved cardiac medications, $18 \%$ involved over the counter medications or dietary supplements, and 13\% involved gastrointestinal medications. Omissions, additions, and dose changes accounted for greater than $75 \%$ of all discrepancies identified in both cohorts with no significant differences between the two cohorts.

The mean number of discrepancies addressed during a TCM visit increased by an average of 6.2 discrepancies per patient from $3.4 \pm 4.1$ in the retrospective analysis to $9.6 \pm 6.3$ in the prospective population with a $p$ value of 0.0003 . The percentage of discrepancies addressed increased by an average of $37.6 \%$ from $26.9 \pm 22.8 \%$ in the retrospective analysis to $64.5 \pm 26.9 \%$ in the prospective population with a $p<0.001$. The percentage of discrepancies addressed remained significantly higher in the post-intervention cohort when stratified by severity as noted in Fig. 1.

\section{DISCUSSION}

Pharmacist involvement in outpatient-based transitions of care significantly increased medication discrepancies addressed by the patient's PCP following discharge from hospital to home. Furthermore, pharmacist involvement led to a significant increase in the percentage of moderate and major discrepancies addressed by the physician. This project's demonstration of an improved medication reconciliation process with pharmacist involvement within a general medicine practice expands the

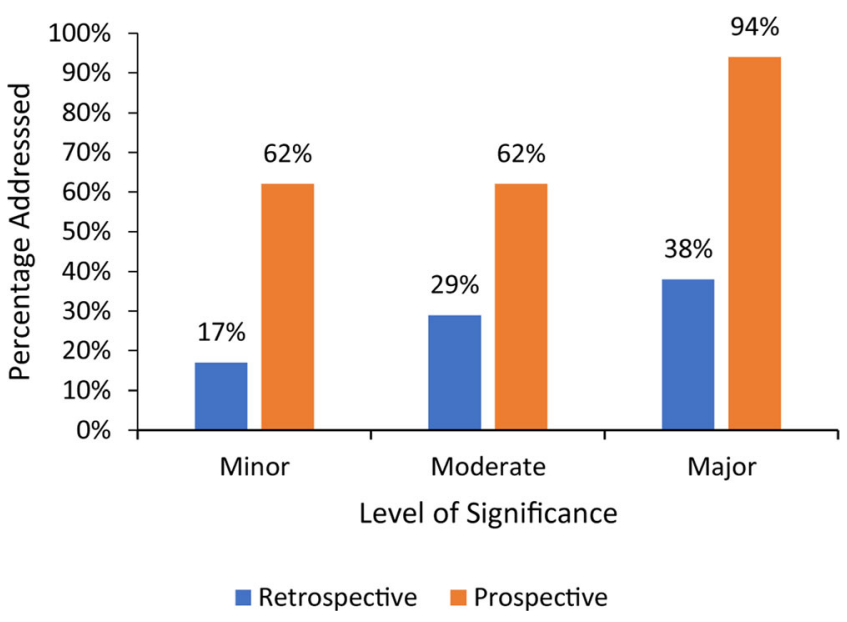

Fig. 1 Percentage of discrepancies addressed stratified by significance

body of evidence that pharmacists improve transitions of care management to include outpatient settings.

Acknowledgements: The authors wish to thank and recognize Jeffery Aeschlimann, PharmD, for his assistance with data analysis and statistical testing.

Corresponding Author: Adam S. Whalley, PharmD; , Portland, USA

Compliance with Ethical Standards:

Conflict of Interest: The authors declare that they do not have a conflict of interest.

\section{REFERENCES}

1. Ploenzke C, Kemp T, Naidl T, Marraffa R, Bolduc J. Design and implementation of a targeted approach for pharmacist-mediated medication management at care transitions. JAPhA. 2016;56:303-9.

2. Schuldt LM. Joint Commission resources: medication reconciliation handbook. 2nd rev. ed. Oakbrook Terrace, IL: Department of Publication Joint Commission Resources; 2009:2-3

3. Mueller SK, Sponsler KC, Kripalani S, Schnipper J. Hospital-based medication reconciliation practices: a systematic review. Arch Intern Med. 2012;172(14): 1057-69

4. Mekonnen AB, McLachlan AJ, Brien JE. Pharmacy-led medication reconciliation programs at hospital transitions: a systemic review and meta-analysis. J Clin Pharm Ther. 2016;41:128-44.

5. Pippins JR, Gandhi TK, Hamann C, Ndumele C, et.al. Classifying and predicting errors of inpatient medication reconciliation. J Gen Intern Med. 2008;23(9):1414-22. 